Supporting Information for

\title{
Surface Strain Induced Collective Switching of Ensembles of Molecules on Metal Surfaces
}

Sha Yang ${ }^{1}$, Shuang Li ${ }^{1}$, Guo-Xu Zhang ${ }^{2}$, Sergey N. Filimonov ${ }^{3}$, Christopher J.

$$
\text { Butch }^{4,5 *} \text {, Ji-Chang Ren }{ }^{1 *} \text {, and Wei Liu }{ }^{1 *}
$$

${ }^{1}$ Nano and Heterogeneous Materials Center, School of Materials Science and Engineering, Nanjing University of Science and Technology, Nanjing 210094, Jiangsu, China.

${ }^{2}$ School of Chemistry and Chemical Engineering, Harbin Institute of Technology, West Dazhi 92, 150001, Harbin, China.

${ }^{3}$ Department of Physics, Tomsk State University, 634050 Tomsk, Russia.

${ }^{4}$ Department of Biomedical Engineering, Nanjing University, Nanjing, China.

${ }^{5}$ Blue Marble Space Institute of Science, Seattle, Washington, 98154, United States.

Corresponding Author. Email: chrisbutch@gmail.com; renj@njust.edu.cn;

weiliu@njust.edu.cn 


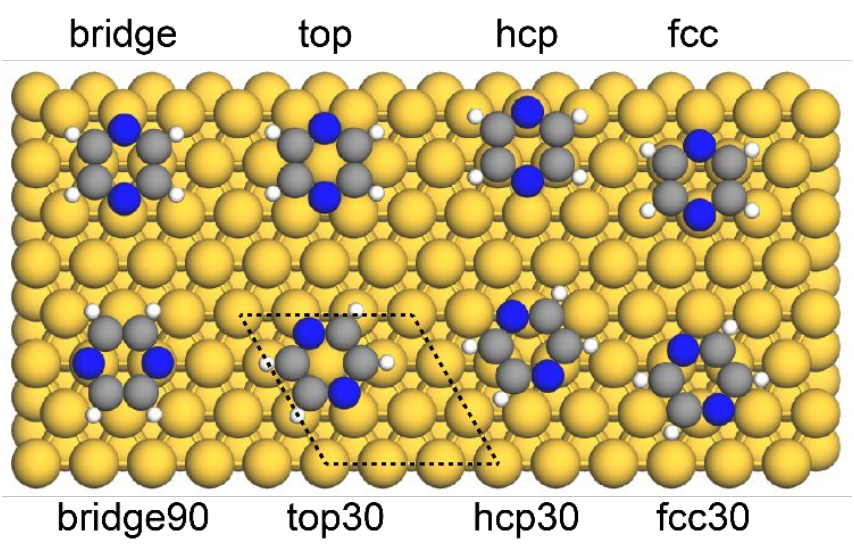

Figure S1 Starting geometries, termed as bridge, bridge90, top, top30, fcc, fcc30, hcp and hcp30 to explore the stable sites. Each site was relaxed at starting adsorption heights of 2.1 and $3.1 \AA$ to ensure the existence of chemisorbed and physisorbed states. Except the significantly smaller adsorption energy at the top30 and bridge 90 sites, energy variation of pyrazine on $\mathrm{Cu}(111)$ at other sites is smaller than $30 \mathrm{meV}$, among which the bridge site is energetically favorable. The dotted line denotes the $3 \times 3 \mathrm{Cu}(111)$ cell. 
(a)

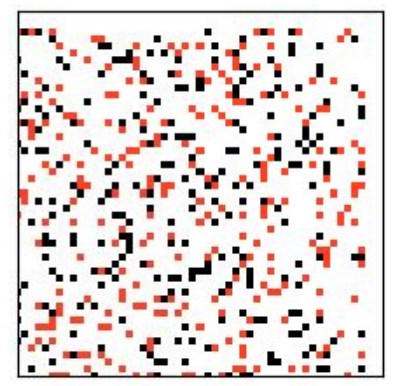

(c)

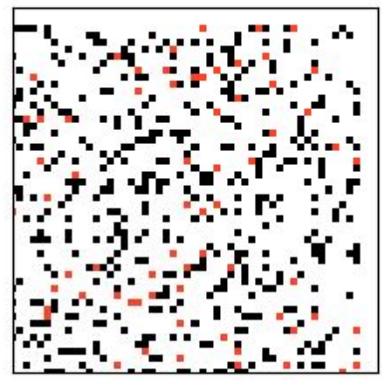

(b)



(d)

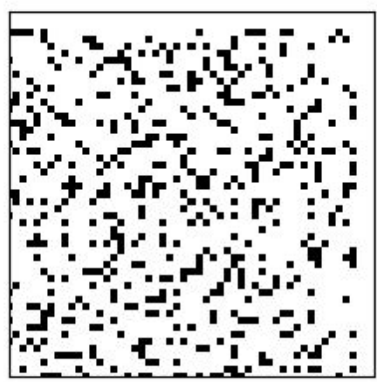

Figure S2 Simulated distributions of the physisorbed state (red) and chemisorbed state (black) by Monte Carlo model. The switching energy barrier was taken as the only variable in the model. Four cases were considered varied by the potential energy surface: equally stable (a), physisorption-favored (b), chemisorption-favored (c), and pure chemisorption (d) cases. 

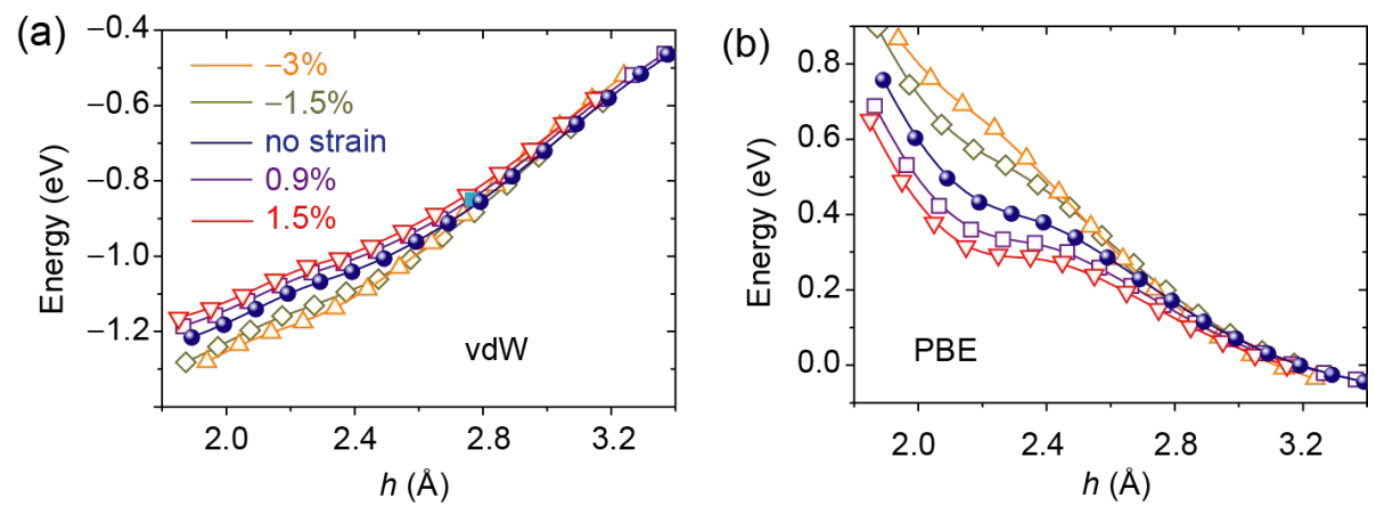

Figure S3 (a) shows the vdW contributions and (b) the PBE contributions decomposed from the binding energies by $\mathrm{PBE}+\mathrm{vdW}$ surf method. The surface strain results in opposing trends in the PBE and vdW contributions. Tensile strain decreases the PBE energies while the vdW energy is increased. The degree that the PBE and $\mathrm{vdW}$ interactions are affected by the strain depends strongly on the adsorption height. For instance, at height of $2.2 \AA$, the strain more significantly influences the PBE than the vdW energies, while at height of $2.8 \AA$ the increase (decrease) in the PBE energy almost exactly equals the decrease (increase) in the vdW energy, leading to nearly constant physisorbed energy. This explains why the strain induces larger changes in the stability of chemisorbed state than physisorbed state. The energy barrier for switching the two states of the contact is thereby overcome through a reversible tensile or compressive surface strain. 




Figure S4 Binding energies of pyrazine molecules on $4 \times 4 \mathrm{Cu}(111)$ (inset) as a function of adsorption distance under varied strains. The intermolecular interactions are negligible (The minimal distance between the atoms of neighboring molecule is $6.0 \AA$ ) for one pyrazine molecule on a $4 \times 4 \mathrm{Cu}(111)$, which, thus, can be seen as a singlemolecule adsorption system. The qualitative effects of strain on the thermal energetics of chemisorption and physisorption are the same at the single-molecule systems with pyrazine physisorption being the favored state on the $\mathrm{Cu}(111)$ under strain of $-3 \%$ while chemisorption is favored under lower compressive strain and tensile strain. This demonstrates that while the exact degree of strain required to change the favored state or achieve barrier-less transition is coverage specific, the qualitative strain-controlled topological tuning is general with respect to molecular coverage. 


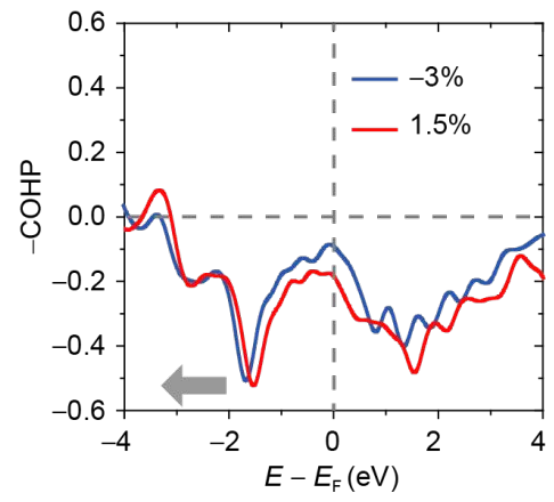

Figure S5 The crystal orbital Hamilton population (COHP) $)^{1-4}$ between the $\mathrm{Cu}$ and $\mathrm{C}$ atoms for the pyrazine/ $\mathrm{Cu}(111)$ system under strain of $-3 \%$ and $1.5 \%$ at a constraint adsorption distance of $2.2 \AA$. Here, the atom-atom $(\mathrm{Cu}-\mathrm{C})$ pairs considered here were limited in a range from 2.0 to $3.6 \AA$. The negative values represent the antibonding states while the positive values denote the bonding states. When applying a compressive strain, the antibonding states near the Fermi level shift to lower energy levels, which leads to higher occupancy. This consequently increases the Pauli repulsion and decreases the stability of the molecules at an adsorption distance of $2.2 \AA$. 
(a)

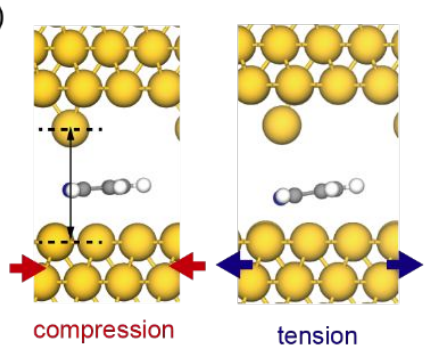

(b)

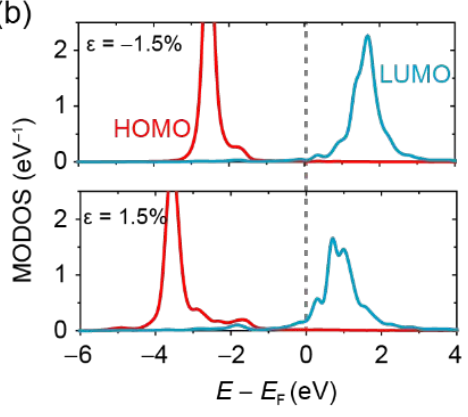

(c)

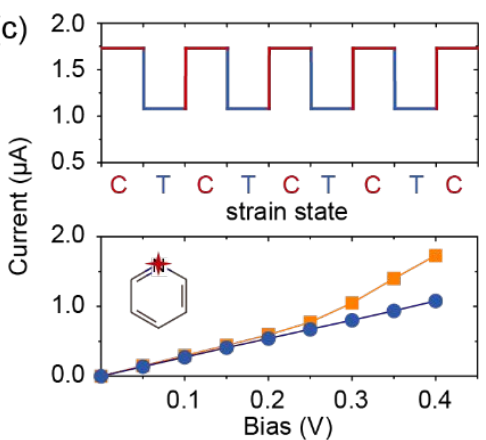

Figure S6 (a) Central regions of pyridine $/ \mathrm{Cu}(111)$ and isoquinoline $/ \mathrm{Cu}(111)$ contacts in NEGF calculations. (b) MODOS of pyridine $/ \mathrm{Cu}(111)$ and isoquinoline $/ \mathrm{Cu}(111)$ systems at compressive and tensile strain conditions. (c) Transport properties of pyridine $/ \mathrm{Cu}(111)$ and isoquinoline $/ \mathrm{Cu}(111)$ contacts. The cross in the lower panel of (c) denotes the tip position. 
(a)

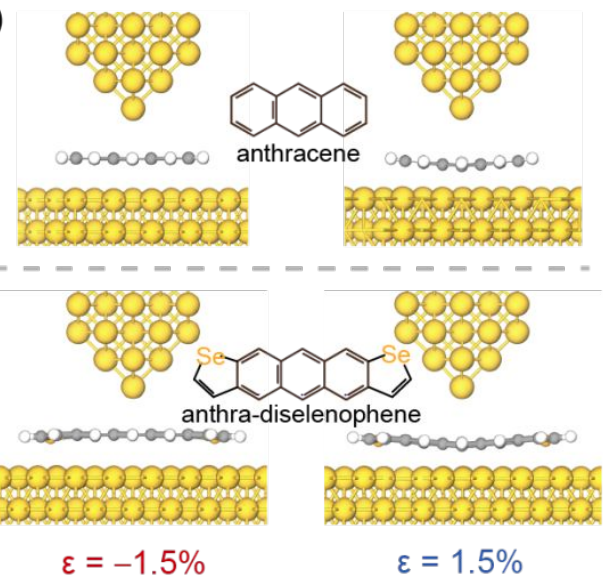

(b)

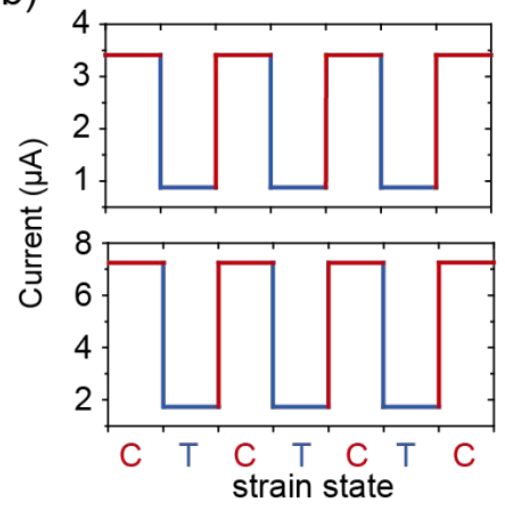

Figure S7 (a) Central regions of anthracene/ $\mathrm{Cu}(111)$ and anthra-diselenophene $/ \mathrm{Cu}(111)$ contacts in NEGF calculations. (b) Transport properties of anthracene/ $\mathrm{Cu}(111)$ and anthra-diselenophene $/ \mathrm{Cu}(111)$ contacts in response to strain. 
(a)

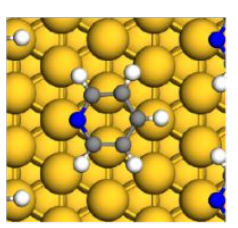

(b)

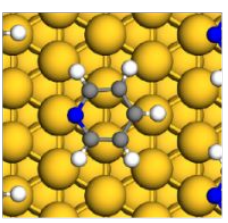

(c)
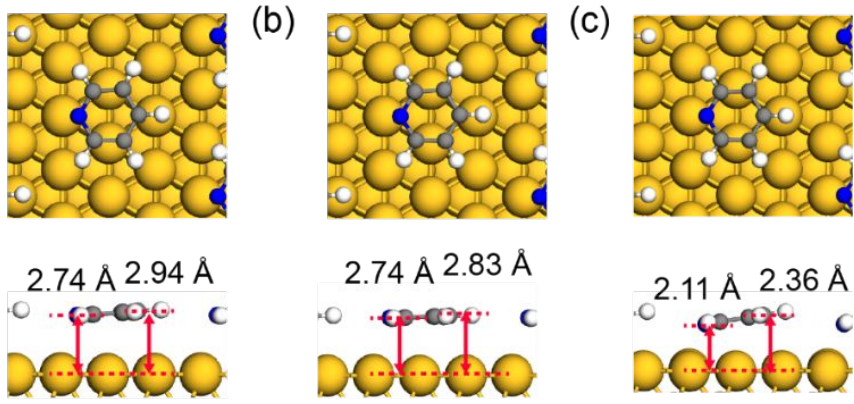

$-1.5 \%$
$2.74 \AA 2.83 \AA$



0

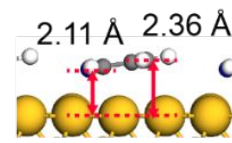

$1.5 \%$

(d)

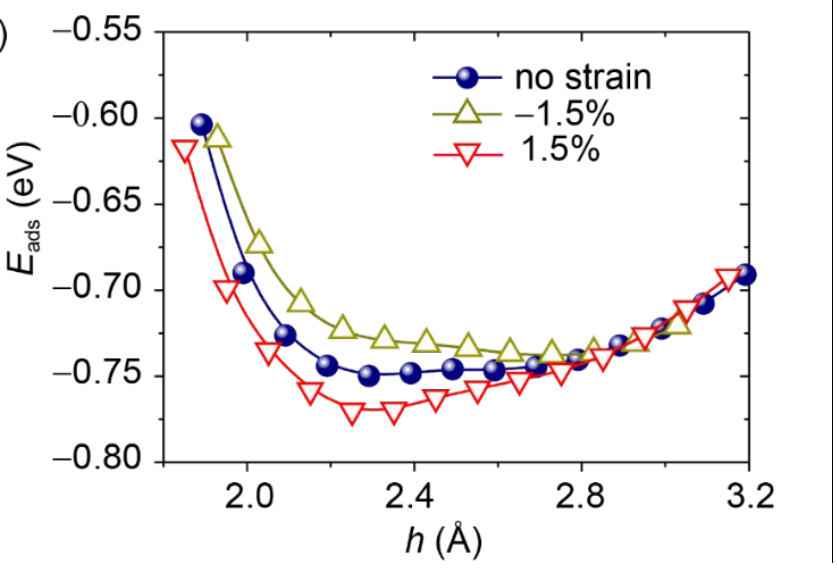

Figure S8 (a)-(c) Adsorption structures of pyridine molecules on $3 \times 3 \mathrm{Cu}(111)$ surface with varied strain. The negative value of strain represents the compressive strain while the positive represent the tensile strain. (d) Binding energies of pyridine on $\mathrm{Cu}(111)$ as a function of the adsorption height of nitrogen atom. The nitrogen atom was constrained during the relaxation while the left atoms were fully relaxed. 
(a)



(b)

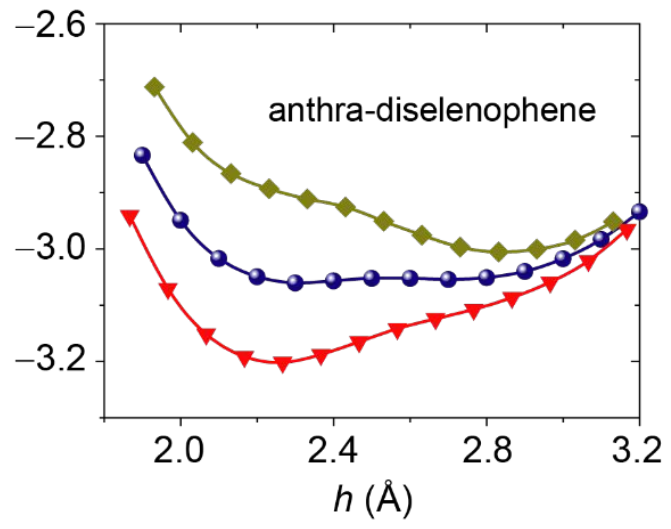

Figure S9 Binding energies of anthracene and anthra-diselenophene on $\mathrm{Cu}(111)$ as a function of the adsorption height of the two central carbon atoms. The two central carbon atoms were constrained during the relaxation while the rest were fully relaxed. 

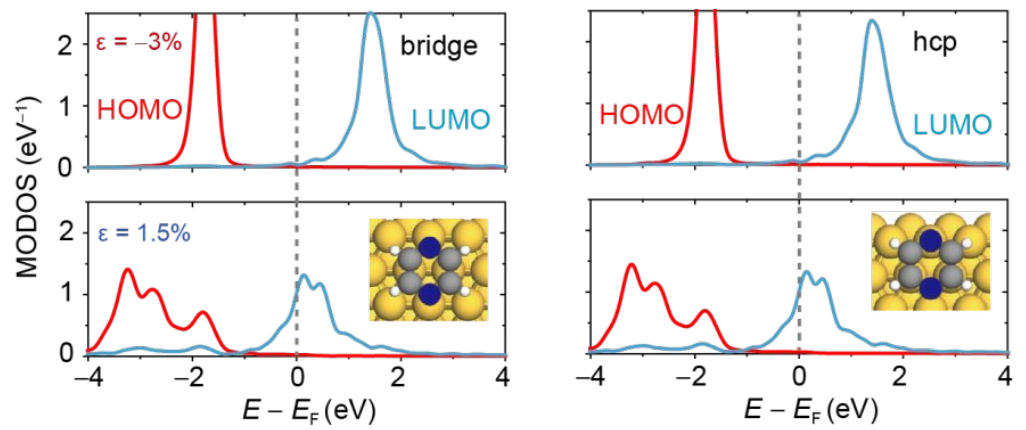

Figure $\mathrm{S} 10$ MODOS of pyrazine on $\mathrm{Cu}(111)$ under $-3 \%$ and $1.5 \%$ strain at the "bridge" and "hcp" sites, respectively. 



Figure S11 (a) Binding energies of pyrazine molecules on $3 \times 3 \mathrm{Cu}(111)$ as a function of adsorption distance under uniaxial strain loading. The uniaxial stress was implemented by applying the strain along the $x$ direction (the green row in the inset) and keeping the $y$ coordinates of the substrate to be constant. The uniaxial stress can be realized by subjecting the polymethylmethacrylate supports covered with a thin $\mathrm{Cu}(111)$ film to uniaxial mechanical loading 5 . (b) Calculated $d$-band center of the $\mathrm{Cu}(111)$ surface as a function of uniaxial (blue) and biaxial (red) stress. As seen in (a), the changes induced by uniaxial stress presents the same trend as biaxial stress in modifying the energies of chemisorption; although there is only slight decrease of potential energy barrier for chemisorption state with tensile strain $1.5 \%$ compared to that with biaxial stress. This is further supported by the lesser slope of the linear relationship between the $d$-band center and the uniaxial strain. 


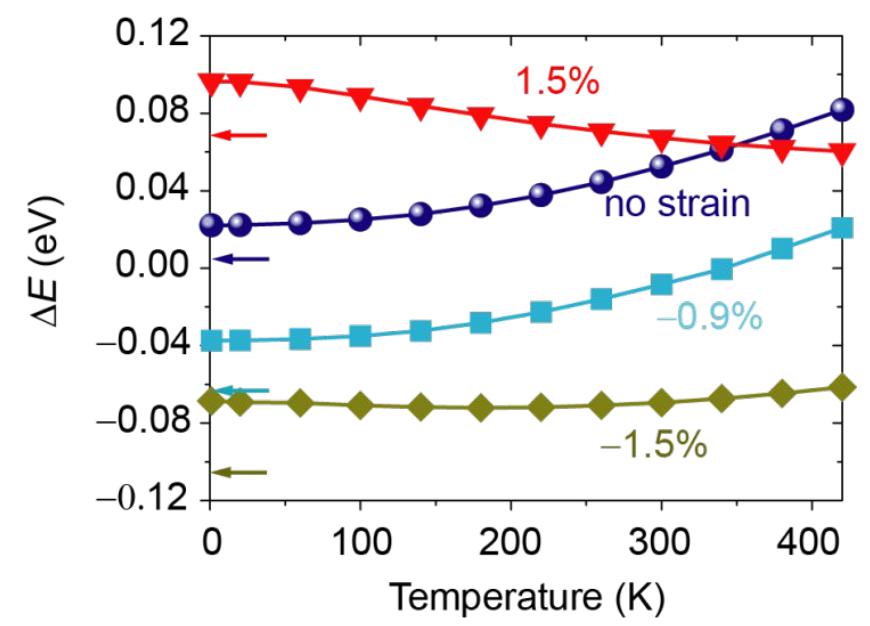

Figure S12 Temperature effect on the energetic stability of pyrazine on $\mathrm{Cu}(111)$ with various strain. The four arrows represent the energy difference between the total energy of chemisorbed and physisorbed systems at $0 \mathrm{~K}$. The metal substrate was fixed at their bulk positions during the calculations. In our thermodynamic calculations, the Gibbs free energy of the adsorption system at temperature $T$ is given by $G_{A}(T)=E_{\text {Total }}+$ $E_{\mathrm{ZPE}}+\Delta U^{\circ}(0 \rightarrow T)-T S^{\circ}(T)$, where $E_{\mathrm{Total}}$ is the total energy determined by DFT calculations, $E_{\mathrm{ZPE}}$ is the zero-point energy, $\Delta U^{q}(0 \rightarrow \mathrm{T})$ the internal energy change from $0 \mathrm{~K}$ to temperature $T$, and $S^{\circ}$ is the standard entropy at temperature $T^{6}$. To identify the temperature effect on the energetics of the interface with various strains we introduce $\Delta G$, defined as the difference of the Gibbs free energies in the physisorbed $G_{\mathrm{A}}$ (phys) and chemisorbed $G_{\mathrm{A}}(\mathrm{chem})$ states. The zero-point energy slightly stabilizes the chemisorbed state $(\sim 0.02 \mathrm{eV})$. The contributions of internal energy and entropy become significant to the interfacial stability as the temperature rises above $300 \mathrm{~K}$. Note that the temperature effect on the interfacial energetic stability is strain dependent. For instance, with tensile strain of $1.5 \%$, increasing the temperature tends to favor the physisorbed state while the temperature effect presents the opposite trend under compressive conditions. 

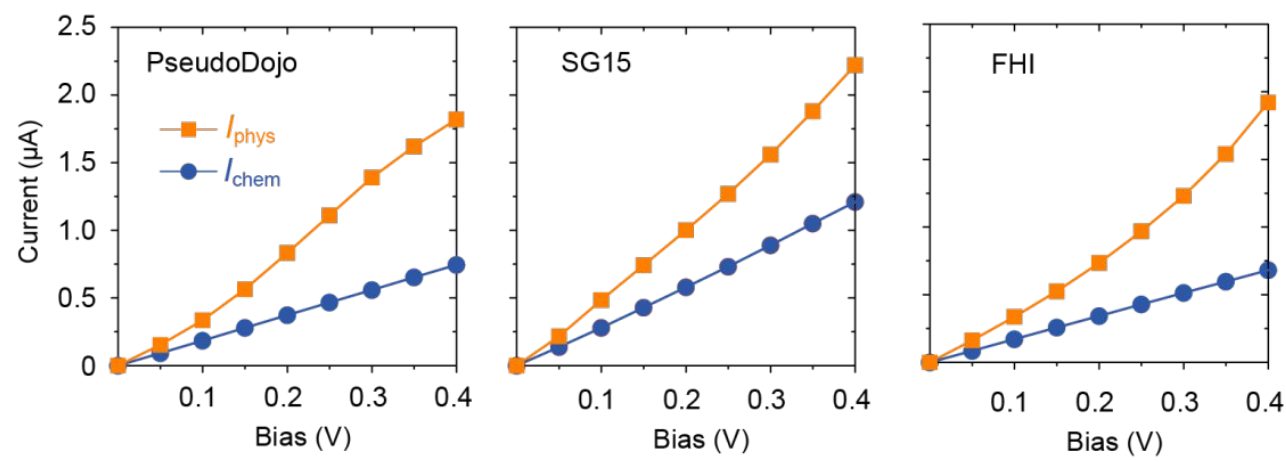

Figure S13 I-V curves of the pyrazine/Cu(111) system under $-3 \%$ and $1.5 \%$ strain conditions calculated by PseudoDojo, SG15 and FHI pseudo-potentials. 


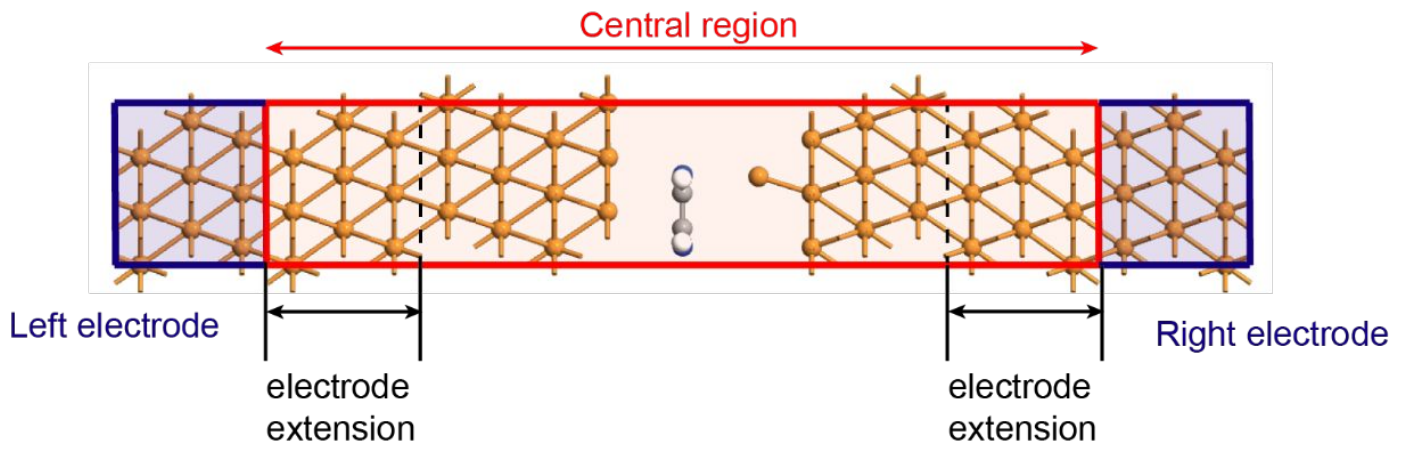

Figure S14 Illustration of the central region and electroded in the transport calculations. The two electrodes are constituted by periodic $\mathrm{Cu}(111)$ surfaces. For the central region, seven layers of metal were used for each side of the central region, while the two electrodes were constituted by periodic $\mathrm{Cu}(111)$ surfaces. In addition, three metal layers (electrode extension) were used at each side to connect the periodic electrodes and the central regions. 



Figure S15 (a) Molecular orbital density of states (MODOS) were determined by projecting the density of states on the HOMO and LUMO for pyrazine on $\mathrm{Cu}(111)$. (b) Projected density of states (PDOS) obtained by projecting the density of states on the whole pyrazine molecule using the same geometries and computational methodology that applied in transport simulations. 

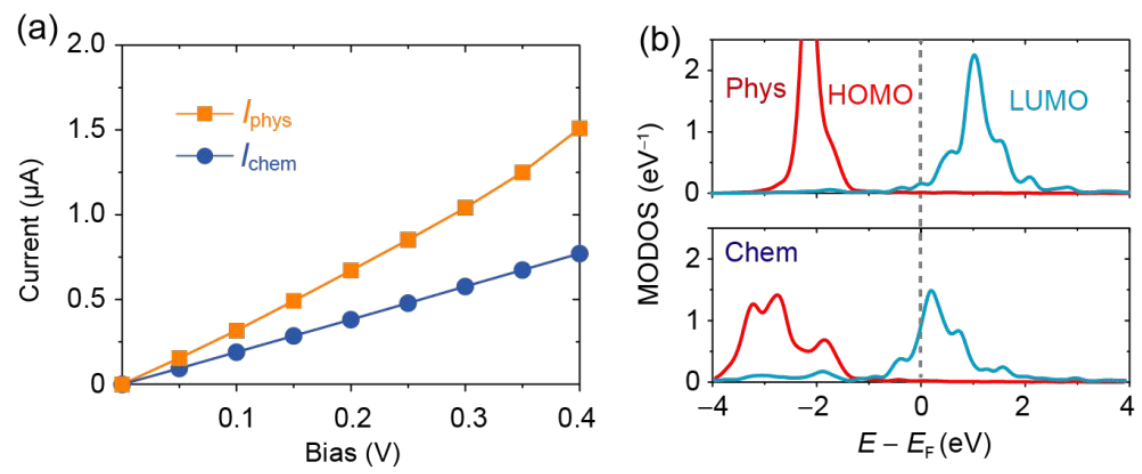

Figure S16 (a) First-principles simulation of $I-V$ characteristics of physisorbed and chemisorbed pyrazine/ $\mathrm{Cu}(111)$ without strain. (b) MODOS of pyrazine/ $\mathrm{Cu}(111)$ physisorbed and chemisorbed states, which were determined by projecting the density of states on the HOMO and LUMO of the free molecule using the same geometry adsorbate has when adsorbed on the surface.

\section{References}

1. Dronskowski R., Blöchl P. E. Crystal orbital Hamilton populations (COHP): energy-resolved visualization of chemical bonding in solids based on densityfunctional calculations. J. Phys. Chem. 97, 8617-8624 (1993).

2. Deringer V. L., Tchougréeff A. L., Dronskowski R. Crystal orbital Hamilton population (COHP) analysis as projected from plane-wave basis sets. J. Phys. Chem. A 115, 5461-5466 (2011).

3. Maintz S., Deringer V. L., Tchougr é eff A. L., Dronskowski R. Analytic projection from plane - wave and PAW wavefunctions and application to chemical - bonding analysis in solids. J. Comput. Chem. 34, 2557-2567 (2013).

4. Maintz S., Deringer V. L., Tchougréeff A. L., Dronskowski R. LOBSTER: A tool to extract chemical bonding from plane - wave based DFT. J. Comput. Chem. 37, 1030-1035 (2016).

5. Yan K., Maark T. A., Khorshidi A., Sethuraman V. A., Peterson A. A., Guduru P. R. The Influence of Elastic Strain on Catalytic Activity in the Hydrogen Evolution Reaction. Angew. Chem. Int. Edit. 55, 6175-6181 (2016).

6. Zhu Y.-A., Chen D., Zhou X.-G., Yuan W.-K. DFT studies of dry reforming of methane on Ni catalyst. Catal. Today 148, 260-267 (2009). 